\title{
A Traveling-Wave-based Fault Location Method in Transmission Line based on LMD and TEO
}

\author{
Qian Hanbo ${ }^{1,2}$ \\ Automation of Department, Nanjing University of \\ Science and Technology, Nanjing Jiangsu Province \\ 210094, China \\ The Center of Coordination \& Support of SASTIND, \\ Beijing 100081, China \\ e-mail: Hanbo.qian@ia.ac.cn \\ Liu Hongbao \\ Industrial and Commercial College, Hebei University \\ Baoding Hebei Province, 071000, \\ e-mail: liuhongbao88@eyou.com
}

\author{
Jia Huibin \\ School of Electrical and Electronic Engineering, North China \\ Electric Power University, Baoding Hebei Province 071003, \\ China; \\ e-mail: hbin.jia@gmail.com
}

\begin{abstract}
Zhang Guoyun
School of Electrical and Electronic Engineering, North China Electric Power University, Baoding Hebei province 071003, China; e-mail: zhangguoyun2013@126.com
\end{abstract}

\begin{abstract}
When Local Mean Decomposition (LMD) is used to detect the arrival time of traveling wave, the instantaneous frequency of the component PF may lead to wrong results for detecting the arrival time of traveling wave. From the above, a novel algorithm is proposed to detect the arrival time of traveling wave, and it is based on Local mean decomposition (LMD) and Teager Energy Operator (TEO). Firstly, the high frequency component of LMD is obtained through decomposing the aerial mode of voltage traveling wave; Secondly, based on Teager Energy Operator(TEO), the instantaneous energy spectrum of PF is calculated; thirdly, the arrival time of traveling wave can be determined according to the first sudden arising of energy in the instantaneous energy of PF1; and the wave velocity can be measured online by calculating the transfer time of traveling wave on the healthy line. Lastly, the fault distance is calculated according to the principle of the double-ended traveling-wave-based fault location. Under different fault conditions, simulation tests show that the novel LMD-TEO-based method has more accurate than Wavelet Transfrom and Hilbert-Huang Transform in detecting the arrival time of traveling wave..
\end{abstract}

Keywords- Traveling wave; Fault Location; Local Mean Decomposition; Teager Energy Operator;Transmission Line

\section{INTRODUCTION}

With the continuous expansion of the scale of power system, the length of transmission line is increasing. When a fault happens in transmission line, a fast and accurate fault location technique is very important to ensure the stable operation of power system. The traveling- wavebased fault location method is considered to get more accurate location and need less time than other fault location methods. Double-ended traveling- wave-based method only needs to detect both ends of the initial traveling wave and has reliable location results, so it is often used for fault location in transmission line[1]. Nevertheless, the accuracy of fault location is still not high because of the impact of the accuracy of detecting poor wave-head, the uncertainty of wave velocity and other factors caused by traveling wave attenuation and distortion. With the rapid development of smart grid and the user requirements to improve the reliability of power system, an accurate traveling-wave-based fault location method has an important significance.

Transient traveling wave produced by the fault of transmission line is a mutant, singularity signal. Compared with other fault location methods, the traveling-wavebased fault location method has higher accuracy. But the transient traveling wave is difficult to be detected because of its short duration and serious attenuation. Wavelet transform is usually to extract the arrival time of traveling wave for fault location [2]. However, the results of Wavelet Transformation (WT) are affected by wavelet basis, sampling rate, decomposition scale and other factors. In theory, wavelet transform has an infinite number of basis functions. If you can not analyze it's the features and characteristics of traveling wave to select the appropriate wavelet basis, it is difficult to obtain satisfactory results [3]. Moreover, wavelet transform is of adjustable window Fourier transformation. Once the scale of wavelet decomposition is decided, the decomposition result is obtained for the signal of a fixed frequency band. The frequency band is only related to the sampling frequency of the signal, and has nothing to do with the signal itself. From this point of view, the wavelet transform has some limitation [4].

Hilbert-Huang Transform (HHT) [5-6] is an adaptive signal decomposition algorithm, and it overcomes the shortcomings of Wavelet Transform which needs select the basic function and decomposition scale. Compared to WT, HHT has a better positioning capability [9].The classical HHT needs to do Empirical Mode Decomposition (EMD) first, and then do Hilbert Transform. However, EMD maybe cause modal mixing phenomenon, so IMF components may not be a single component signal; and 
when it is used for detecting the arrival time of traveling wave, it is very difficult to determine the accurate arrival time of traveling wave. Local Mean Decomposition (LMD) is a new time-frequency analysis method, and it is an adaptive signal decomposition one. But LMD algorithm has end effects and modal mixing phenomenon. If we use instantaneous frequency of $P_{F}$ component to detect the arrival time of traveling wave, which may cause inaccurate detection and even fails to detect. Although some literatures propose several methods to eliminate mode mixing phenomena [10-12], it can not be completely eliminated; it can only be reduced to a certain extent.

When traveling wave arrives, large fluctuations in the frequency and amplitude of the signal happen. Teager Energy Operator (TEO) [13-15] can effectively reflect the signal change of amplitude and frequency. The energy value of TEO can effectively detect the singular position of the analyzed signal. Therefore, we can detect TEO's energy value of traveling wave to determine the arrival time of traveling wave.

Based on the above analysis, a combination of Local Mean Decomposition and Teager Energy Operator(Referred as LMD-TEO)is proposed to determine the arrival time of traveling wave to improve fault location accuracy.

In this paper, LMD-TEO is introduced firstly; secondly, LMD-TEO applied to traveling-wave-based fault location is described; thirdly simulation is tested under different cases such as different fault initial angle, different length line, different fault resistance etc. Experimental test shows that the method proposed in this paper obtains the more accurate than Wavelet Transform, classical HHT, and LMD method. Lastly, the conclusion is given.

\section{ALGORITHM OVERVIEW}

\section{A. Local Mean Decomponent}

Local Mean Decomposition (LMD) is a new adaptive time-frequency analysis method which is proposed by Jonathan S.Smith. It can decompose a complex nonstationary signal into the sum of several $P_{F}$ components. Those $P_{F}$ of LMD can save more frequencies and envelope information than IMF of EMD and LMD method also has fewer iterations, faster computing speed, and thus its end effect is obvious. Energy of Timefrequency analysis based on LMD is more focused than it is in Hilbert spectrum[12]. The phenomenon of negative frequency in Hilbert transform which can not be explained is also avoid. For any given signal $\mathrm{x}(\mathrm{t})$, the process of LMD method is as follows:

1)Find all local extrema $n_{i}$ of $\mathrm{x}(\mathrm{t})$ and calculate the local mean function $m_{11}(\mathrm{t})$ and envelope estimation function $a_{11}(\mathrm{t})$.Set any two adjacent extreme points of $n_{i}$ and $n_{i+1}$,There are:

$$
\begin{aligned}
& m_{i}=\left(n_{i}+n_{i+1}\right) / 2 \\
& a_{i}=\left|n_{i}+n_{i+1}\right| / 2
\end{aligned}
$$

2)Average value $m_{i}$ and envelope Estimate value ${ }^{a_{i}}$ were connected with a line smoothing, we can get local mean function $m_{11}(\mathrm{t})$ and envelope estimation function ${ }^{a_{11}}(\mathrm{t})$ 。
3 ) Calculation of the envelope estimation function $a_{1 n}(\mathrm{t})$ and frequency modulation function $s_{1 n}(\mathrm{t})$.The local mean function $m_{11}(\mathrm{t})$ separate from the original signal $\mathrm{x}(\mathrm{t})$, and we have:

$$
\begin{aligned}
& h_{11}(t)=x(t)-m_{11}(t) \\
& s_{11}(t)=h_{11}(t) / a_{11}(t)
\end{aligned}
$$

If $a_{12}(\mathrm{t}) \neq 1, s_{11}(\mathrm{t})$ will be regarded as the original data to repeat the iterative process until ${ }^{a_{1(n+1)}(\mathrm{t})}=1$. And $\mathrm{S}_{1 n}(\mathrm{t})$ is a FM signal, the envelope signal is

$a_{1}=a_{11}(\mathrm{t}) a_{12}(\mathrm{t}) \cdots a_{1 n}(\mathrm{t})=\prod_{i=1}^{n} a_{1 i}(\mathrm{t})$

4 ) Calculate the $P_{F}$ component. The envelope signal $a_{1}(\mathrm{t})$ and the pure frequency modulation signal function, $\mathrm{s}_{1 n}(\mathrm{t})$ is multiplied to obtain the first $P_{F}$ component of the original signal:

$$
P_{F 1}(\mathrm{t})=a_{1}(\mathrm{t}) \mathrm{S}_{1 n}(\mathrm{t})
$$

Separate the first $P_{F}$ component from original signal $\mathrm{x}(\mathrm{t})$, and get a new signal $\boldsymbol{u}_{1}(t)$.The $\boldsymbol{u}_{1}(t)$ will be regarded as the original data and repeat the above steps and cycle $\mathrm{k}$ times until $\boldsymbol{u}_{k}(t)$ is a monotonic function.The original signal is decomposed into a sum of $P_{F}$ components and a monotonic function $u_{k}(t)$ :

$$
\mathrm{x}(\mathrm{t})=\sum_{i=1}^{k} P F_{i}(t)+u_{k}(t)
$$

$P_{F}$ component is combined with instantaneous frequency of PF component, the complete time-frequency distribution of original signal $x(t)$ can be obtained. The envelope signal $a_{k}(\mathrm{t})$ is the instantaneous amplitude signal and the signal frequency can be obtained from the pure frequency modulation signal $s_{k n}(\mathrm{t})$.Document [13] use the direct method to calculate the instantaneous frequency of the signal. The method is simple and direct, but there exist some deviation when $s_{k n}(\mathrm{t})= \pm 1$. For multi-component signal, a multi-component signal could have multiple frequencies at any time. So there are certain limitations to calculate the instantaneous frequency of the signal directly with the phase of the derivative. For this purpose, we can use other methods to detect a single-component signal which LMD method.

\section{B. Teager Energy Operator}

Teager Energy Operator(TEO) is a nonlinear one, which can effectively extract the signal energy, and can reflect the instantaneous energy change. For the case of continuous-time signal $\mathrm{s}(\mathrm{t})$, TEO is defined as:

$$
\psi_{d}[\mathrm{~s}(\mathrm{t})]=s^{\prime 2}(\mathrm{t})-s(t) \mathrm{s}^{\prime \prime}(\mathrm{t})
$$

Where $\mathrm{s}^{\prime}(\mathrm{t})$ is $\mathrm{s}(\mathrm{t})$ derivative; $\Psi$ is energy operator. for real discrete-time signal:.

$$
\psi[\mathrm{s}(n)]=s^{2}(n)-s(n-1) \mathrm{s}(n+1)
$$

Teager energy operator algorithm was an approximate transform of mathematical expression, whose principle was simple, without complex calculations, It can also follow signal changes quickly. The more rapidly amplitude or frequency of a signal changes, 
the greater energy value calculated by TEO. Based on the characteristics, and it had been widely used in detecting the singularity of the signal.

\section{PRINCIPLE OF DETECTING THE ARRIVAL TIME OF TRAVELING WAVE}

When traveling wave reaches bus bar, the highfrequency components of traveling wave has a large fluctuation of amplitude and frequency, i.e. a sudden change of instantaneous frequency. In this paper, the voltage signal of traveling wave is selected for fault location and Karrenbauer transformation is used to decouple the voltage signal of traveling wave. After the intrinsic mode component $P_{F}$ is obtained by decomposing the decoupled traveling wave, TEO is used to calculate instantaneous energy value of $P_{F 1}$ component. When traveling wave arrive test points, it shows great change in frequency and amplitude, the instantaneous energy value of Teager changes, too. So we can determine the arrival time of traveling wave by obtaining the corresponding time of the maximum point of TEO.

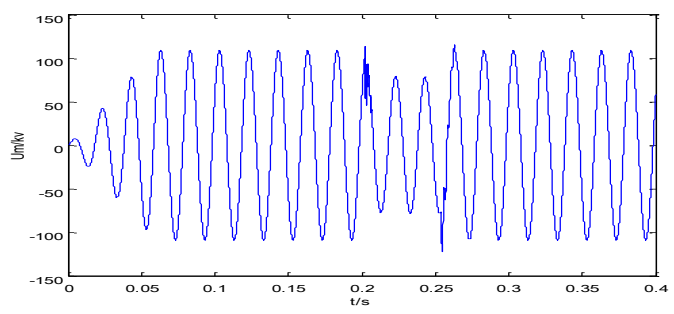

Figure 1. a -mode traveling wave

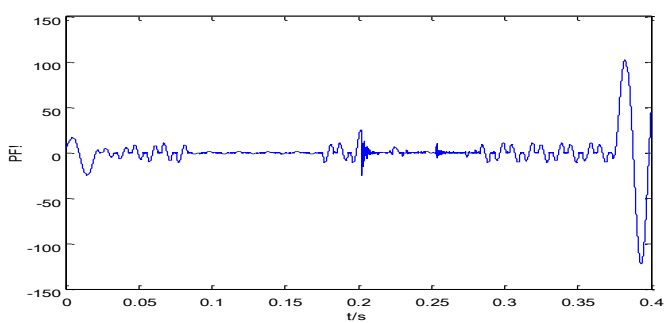

Figure 2. PF1 component

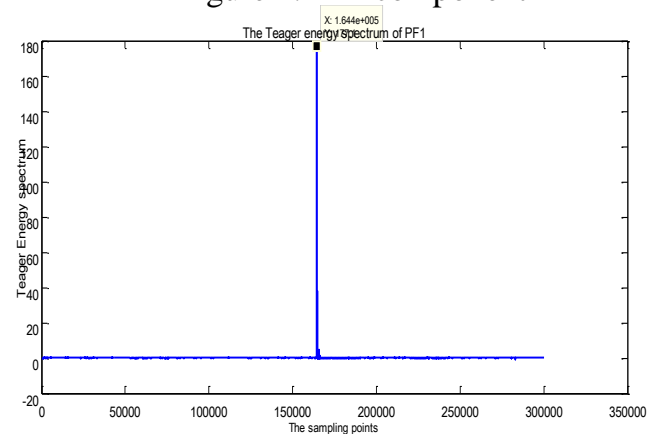

Figure 3. Teager energy spectrum of PF1

When A-phase-ground fault happens via $100 \Omega$ resistor. The fault occurs at $0.0164 \mathrm{~s}$ and a corresponding fault traveling wave happens. The a -mode voltage of traveling wave is shown in Fig.1, and PF1 of its LMD decomposition is shown in Fig.2.

TEO is used to calculate the instantaneous energy spectrum of $P_{F 1}$, which is shown in Fig.3. As can be seen from Fig.3, the first sudden energy point of $P_{F}$ instantaneous energy corresponds to the arrival time of the initial fault traveling wave [16-18]. Compared to the transient spectrum of $P_{F 1}$, the arrival time of the initial traveling wave is more obvious in instantaneous energy spectrum. So the corresponding time of TEO's maximum value can be used as the arrival time of the initial traveling wave.

\section{SIMULATION AND ANALYSIS}

\section{A. Simulation Model}

Electromagnetic Transient Program PSCAD/EMTDC is used to simulate electromagnetic transient fault.

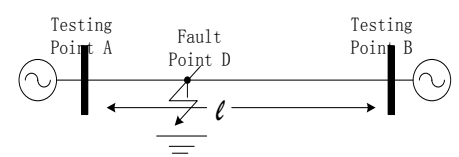

Figure 4. The transmission lines of simulation Table 1 The positive sequence and zero sequence

\begin{tabular}{ccccc}
\hline $\begin{array}{c}\text { Parameter } \\
\text { Type }\end{array}$ & $\mathrm{r}(\Omega / \mathrm{km})$ & $\mathrm{x}(\Omega / \mathrm{km})$ & $\mathrm{g}(\mathrm{s} / \mathrm{km})$ & $\mathrm{b}(\mathrm{s} / \mathrm{km})$ \\
\hline $\begin{array}{c}\text { Positive } \\
\text { parameter } \\
\text { Zero }\end{array}$ & 0.0407815 & 0.3079 & $2.7 \times 10^{-6}$ & $3.7 \times 10^{-6}$ \\
parameter & 0.205528 & 0.69188 & $2.7 \times 10^{-6}$ & $2.19 \times 10^{-6}$ \\
\hline
\end{tabular}

Test points are placed at both ends of transmission line, the sampling frequency is set to $1 \mathrm{MHz}$ (The sampling period is $1 \times 10-6 \mathrm{~s})$. Short-circuit fault happens at $20 \mathrm{~km}$ 、 $60 \mathrm{~km} 、 110 \mathrm{~km} 、 140 \mathrm{~km}$ from test point A. In each case, the transition resistance is $20 \Omega, 50 \Omega, 100 \Omega$, and $300 \Omega$, respectively. The initial angle is set to $90^{\circ}, 60^{\circ}, 45^{\circ}, 30^{\circ}$, respectively. Short-circuit fault happens at $0.164 \mathrm{~s}$.

Karrenbauer transformation is used to decouple the voltage traveling wave. Then the voltage traveling wave of $\alpha$-model is used for LMD decomposition, and TEO is used to calculate the instantaneous energy spectrum of $P_{F 1}$ component. The corresponding time of TEO's maximum value is the arrival time of the initial traveling wave. In order to avoid the impact of velocity, the velocity needs to be calculated online. In this paper, the velocity online is $\mathrm{v} \approx 2.9935403 \times 108 \mathrm{~m} / \mathrm{s}$.

\section{B. Analysis of fault location}

In this chapter, a simulation model of single phaseground fault in transmission lines is built by using the PSCAD / EMTDC and then simulation data is obtained in Matlab software. The double-ended traveling-wave-based method is used for fault location. Simulation results show the feasibility of the fault location method based on LMDTEO.

\section{1) Simulation results under different fault type}

Due to space limitations, only four classical fault types are adopted for testing the method proposed in the paper. The fault happens at $60 \mathrm{~km}$ away from testing point $\mathrm{A}$ when the transition resistance is $100 \Omega$. Simulation results are shown in Table 2. 
Table 2 Error under different fault type

\begin{tabular}{ccc}
\hline fault type & $\begin{array}{c}\text { distance } \\
(\mathrm{km})\end{array}$ & error $(\mathrm{km})$ \\
\hline $\mathrm{Ag}$ & 60.064 & 0.064 \\
$\mathrm{AC}$ & 60.064 & 0.064 \\
$\mathrm{ACg}$ & 59.914 & 0.085 \\
$\mathrm{ABC}$ & 60.064 & 0.064 \\
$\mathrm{ABCg}$ & 59.914 & 0.085 \\
\hline
\end{tabular}

2) Simulation results under different initial angles

When a fault happens, the initial angle directly affects the amplitude of the transient voltage of traveling wave [18].To study the effects of different initial angle, the initial angle is set to $90^{\circ}, 60^{\circ}, 45^{\circ}, 30^{\circ}$ and a singleto-ground fault happens at $60 \mathrm{~km}$ away from testing point A. The simulation results are shown in Table 4.

Table 4 Error under different initial angles

\begin{tabular}{ccc}
\hline initial angle $/{ }^{\circ} \mathrm{C}$ & distance $/ \mathrm{km}$ & error $/ \mathrm{km}$ \\
90 & 60.064 & 0.064 \\
60 & 60.064 & 0.064 \\
45 & 59.91 & 0.09 \\
30 & 59.91 & 0.09 \\
\hline
\end{tabular}

3) Simulation results under different transimition resistances

When a fault happens, transition resistance has a direct influence on the amplitude of traveling wave. To test the method proposed in this paper, the transition resistance is set to $20 \Omega, 50 \Omega, 100 \Omega$, and $300 \Omega$ when a single-toground fault happens at $60 \mathrm{~km}$ away from testing point A. Simulation results are shown in Table 5.

Table 5 Error at different transition resistances

\begin{tabular}{ccc} 
resistance $/ \Omega$ & distance $/ \mathrm{km}$ & error $/ \mathrm{km}$ \\
20 & 60.064 & 0.064 \\
50 & 60.064 & 0.064 \\
100 & 60.064 & 0.064 \\
300 & 59.91 & 0.09 \\
\hline
\end{tabular}

4) Simulation results under different location algorithms

In order to test the method proposed in this paper, $t$ wavelet (db3), HHT and LMD are used for fault location under different conditions. The simulation results are shown in Table 6.

Table 6 Error analysis of different location methods

\begin{tabular}{cccc}
\hline Distance from & Location & Distance $/ \mathrm{km}$ & Error $/ \mathrm{km}$ \\
Testing Point $(\mathrm{km})$ & Algorithm & & \\
20 & Wavelet & 20.4 & 0.4 \\
& HHT & 20.25 & 0.25 \\
& LMD & 20.1 & 0.1 \\
& LMD-TEO & 19.951 & 0.049 \\
\hline
\end{tabular}

\begin{tabular}{cccc}
\hline \multirow{2}{*}{110} & Wavelet & 110.356 & 0.356 \\
HHT & 110.209 & 0.209 \\
LMD & 109.90 & 0.092 \\
LMD-TEO & 110.056 & 0.056 \\
\hline
\end{tabular}

From the Table 6, it is shown that LMD-TEO method can obtain more accuracy than wavelet, HHT and LMD when they are used for fault location.

\section{CONCLUSION}

When Local Mean Decomposition (LMD) is used to detect the arrival time of traveling wave, the instantaneous frequency of the component PF may lead to wrong results for determining the arrival time of traveling wave. In this paper, a novel algorithm is proposed to detect the arrival time of traveling wave, and it is based on Local mean decomposition (LMD) and Teager Energy Operator (TEO). The wave velocity can be measured by calculating the transfer time of traveling wave on the healthy line. The fault distance is calculated according to the principle of the double-ended traveling-wave-based fault location. Under different fault conditions, simulation tests show that the novel LMD-TEO method has more accuracy than Wavelet Transform and Hilbert-Huang Transform in detecting the arrival time of traveling wave.

\section{ACKNOWLEGEMENT}

This paper is granted by Natural Science Foundation of Hebei Province (E2013502267) and the Fundamental Research Funds for the Central Universities (13MS68).

\section{REFERENCES}

[1] QIN Jian. Study on single terminal traveling wave fault location of transmission line[J]. Power System Technology, 2005, 29(15): 65 70 .

[2] ZHENG Zhou, LÜ Yan-ping, WANG Jie, WU Fan. A new twoterminal traveling wave fault location method based on wavelet transform [J] Power System Technology; 01, 2010

[3]

[4] Han xu.Research the fault location of power systems with traveling wave based on Hilbert-Huang transform[D] Yanshan university, 2010

[5] Zhong Youming, Qin Shuren, Tang Baoping. Study on a new transformation method for vibration[J]. Journal of vibration engineering, 2002, 15 (2) : 233-238.

[6] Huang $\mathrm{N}$ E, shen Z, Long S R, et al. The empirical mode decomposition and the Hilbert spectrum for nonlinear and nonstationary time series analysis[J]. Proc.R SocLond A, 1998, 454 (21) : 903-995.

[7] Huang N E, Shen Z, Long S R.Anew view of nonlinear waves: the Hilbert spectrum[J]. Annual Review of Fluid Mechanics, 1999, 31: 417-457.

[8] MA Dan-dan, WANG Xiao-ru. Single terminal methods of traveling wave fault location based on wavelet modulus maxima [J]. Power System Protection and Control , 2009, 37(3): 55-59 .

[9] LIANG Jin, ZHOU Jing. Application of multiwaves theory for the fault location in transmission line $[\mathrm{J}]$. Power System Protection and Control, 2008, 36(13): 26-32.

[10] ZHANG Xiaoli,ZENG XiangJun,MA HongJiang,LI Zewen. Power grid faults location with traveling wave based on Hilbert-Huang Transform $[\mathrm{J}]$. Automation of Electric Power Systems, 2008, 32(8):64-68. 
[11] Smith J S. The local mean decomposition and its application to EEG perception data[J]. Journal oft he Royal Society InterFace, 2005, 2(5): 443-454.

[12] LIN Wanru, XIONG Shengwu, XIE Xiaohu. Partial empirical model decomposition[J]. Computer Engineering and Applications, 2011, 47(3): 123-126.

[13] Zhu Yongli Fan Xinqiao Yin Jinliang,A new fault location scheme for transmission lines based on traveling waves of three measurements[J]. Journal of electrical engineering technology, 2012 (3) : 260-268.

[14] Ren Daqian. Study on methods and system for fault characteristics extraction of rotating machines based on local mean Decomposition[D]. Zhejiang: zhejiang university, 2008.

[15] Teager H M. Some observations on oral air flow during phonation[J]. IEEE Transactions on Acoustics, Speech,and Signal Processing, 1980, 28 (5): 599-601.
[16] Maragos P, Kaiser J F, Quatieri T F. Energy separation in signal modulations with applications to speech analysis[J]. IEEE Transactions on Signal Processing, 1993, 41 (10): 3024-3051.

[17] Fan Xinqiao, Zhu Yongli Lu Weifu. Traveling wave based fault 10cation for transmission lines based on EMD-TEO[J]. Power system protection and control, 2012, V40 (9) : 8 to 12 in 2.

[18] Wu Hao,Xiao Xianyong,Deng Wujun.Identification of lightning strike and fault in the traveling wave location of transmission line[J]. High voltage technology, 2007, 33 (6) : 63-67.

[19] ZENG Xiang-jun,ZHANG Xiao-li,MA Hong-jiang,LEI Li. Traveling wave fault location method for power grids based on wavelet packet energy spectra[J]. High voltage technology, 2008, 34 (11) : 2311-2316 